\title{
Drainage of pyogenic sacro-iliac joint infection using a percutaneous technique (case series of 13 patients)
}

\begin{abstract}
Purpose:Pyogenic sacro-iliac joint (SIJ) infection is a rarely diagnosed musculo-skeletal infection. Symptoms and signs are commonly non-specific with a vague clinical picture and poorly defined localization of symptoms. This usually results in low clinical suspicion. This fact causes diagnosis to be difficult and delayed for several weeks or months.
\end{abstract}

Methods: This study included 13 patients with the confirmed diagnosis of SIJ pyogenic infection who needed drainage after failure to respond to conservative treatment. The study was carried out between February 2012 and November 2015. Once suspected of having SIJ infection, patients where sent for radiographic and Laboratory tests. The drainage procedure was performed in the operative theatre using local infiltration anesthesia and under conscious sedation.

Results: The mean age of patients was 32 . The mean time interval from initial complaint till diagnosis was 12 weeks. C-reactive protein and ESR levels were at least double the normal in all 13 patients. Radiological changes in the form of SIJ destruction/sclerosis (x-ray, CT) were evident in 10 of the 13 patients. MRI evidence of SIJ infection was noted in all 13 patients. Mean improvement in VAS, ODI at end of treatment was 5.5, 64 respectively. Complications occurred in 4 patients and included, pelvic abscess formation, ilio-psoas abscess formation, posterior subcutaneous abscess, septicemia, SIJ instability

Conclusion: When conservative treatment fails or presentation is late with abscess formation and /or bone destruction, minimally invasive image guided drainage and irrigation can be very useful and will have minimal morbidity.

Keywords: Infection, Percutaneous, Pyogenic, Sacro-iliac joint
Volume 8 Issue 2 - 2017

\author{
Ahmed Mohamed Elbadrawi,Ahmed \\ Mohamed Morsi,Tameem Mohamed \\ Elkhateeb \\ Department of Orthopedics and Spine Surgery, Faculty of \\ Medicine, Ain Shams University, Egypt
}

Correspondence: Tameem Mohamed Elkhateeb, Department of Orthopedics and Spine Surgery, Faculty of Medicine, Ain Shams University, Nasr City, Cairo, Egypt, Tel (+20 I00) 1809052 Fax (+20100) 1809052, Email tameen_77@hotmail.com; tameen_elkhateeb@med.asu.edu.eg

Received: March 06, 2017 | Published: April 27, 2017

\section{Introduction and background}

Pyogenic sacro-iliac joint (SIJ) infection is a rarely diagnosed musculo-skeletal infection; it accounts for 1-2\% of septic arthritis and osteomyelitis. ${ }^{1-3}$ Symptoms and signs are commonly non-specific with a vague clinical picture and poorly defined localization of symptoms. Moreover, the clinical presentation can mimic many disorders of nearby joints and bones. Differential diagnosis includes disorders of hips joints, lumbo-sacral spine, and even other intra-pelvic and visceral pathology. This usually results in low clinical suspicion. This fact causes diagnosis to be difficult and delayed for several weeks or months. ${ }^{4,5}$

Haematogenous spread of pathogens through the prevertebral and paravertebral plexus of veins, reaches the anterior aspect of the sacroiliac joint (SIJ) where vascularity is high. ${ }^{3}$ The iliac side of the joint is more commonly affected. A possible explanation is the fact that there is a thicker cartilage barrier covering the sacral side of the joint. ${ }^{6,7}$

Most reported cases are children and young adults. Still, SIJ infection can occur in any age group. Males are as twice affected as females. The most frequently isolated pathogen is Staph. aureus, but streptococci, E. coli, Mycobacterium tuberculosis and Salmonella have also been isolated. ${ }^{8}$

High index of suspicion is needed for early diagnosis. Risk factors that need to be observed include: IV drug abuse, immune incompetence, and nearby or recent pyogenic infections. ${ }^{3}$ Once Pyogenic SIJ infection is suspected, the patient should be hospitalized. ${ }^{9}$
Treatment of pyogenic SIJ infection depends on the stage of presentation and response to antimicrobials. In early stages without bone destruction, parenteral followed by oral antimicrobials will usually control the infection. When diagnosis is delayed, which is more commonly seen, treatment with antibiotics alone is often unsuccessful in osteomyelitis or arthritis of the pelvis. When pus is formed, image guided joint aspiration should be done and the aspirate is then cultured to identify the pathogen (successful in $80 \%$ of cases) and drainage (with or without fusion) will usually be needed. ${ }^{4,9}$

\section{Patients and methods}

This study included 13 patients with the confirmed diagnosis of SIJ pyogenic infection who needed drainage after failure to respond to parenteral antibiotics and bed rest. The study was carried out between February 2012 and November 2015. Once suspected of having SIJ infection, patients where sent for Plain radiographs, MRI, CT, and Laboratory tests: ESR, CRP (quantitative), and WBC count. Blood cultures were ordered when patients had fever. Patients were also examined and investigated for predisposing factors and pre-existing potential sources of infection.

Patients were examined for tenderness/swelling over the SIJ, and whetherSIJ stresstests(Gaenslen'stest:hiphyperextension;FABERtest: hip flexion-abduction-external rotation) caused or increased pain over the SIJ. ${ }^{10}$ Hip pathology was excluded by range of motion examination and the lumbar spine was locally examined together with femoral and sciatic stretch tests and neurological examination of both lower limbs. When a source of infection was identified and was more accessible 
than the SIJ, samples were obtained and cultured. When no primary source was identified, C-arm image guided aspiration and drainage of the SIJ were performed under local anesthesia and conscious sedation, and the aspirates were microscopically examined on stained films, and were cultured on different bacterial media (aerobes, anaerobes and specific culture media).

\section{Aspiration and drainage technique}

The drainage procedure was performed in the operative theatre using local infiltration anesthesia from skin down to bone and under conscious sedation. Patients were positioned in the supine position over the orthopedic table allowing $\mathrm{C}$-arm use in both anterior and lateral projections.

A small stab incision was made over the lateral aspect of the posterior ilium. A $0.8 \mathrm{~mm}$ guide wire is initially introduced using the true lateral C-arm projection which was confirmed by perfect overlap of both greater sciatic notches. The entry point of the guide wire should always be below the projection of the pelvic inlet. Using a drill, the guide wire is inserted at the outer table of the iliac wing and is advanced through bone pointing towards the superior sacrum. The position of the wire is then verified using anterior $\mathrm{C}$-arm projection. A draining metallic cannula (outer diameter $3.5 \mathrm{~mm}$, inner diameter $=$ $2.5 \mathrm{~mm}$ ) is then introduced manually and with gentle tapping through bone along the guide wire till it enters the joint. The guide wire is then removed and aspiration and drainage of any collection is then carried out, followed by repeated irrigation and suction using normal saline and gentamycin. Aspirated samples were sent for microscopic examination, culture and anti-biotic sensitivity.

Patients were mobilized as their pain permitted. Follow up and timing of shifting to oral antibiotics as well as their discontinuation was determined based on serological testing (ESR, CRP, WBC count).

Clinical response to drainage followed by antibiotic treatment was evaluated using improvements in visual analogue scale (VAS) and Oswestry disability index (ODI). These were measured on hospital admission, 2 days after drainage, and when laboratory tests returned to normal.

\section{Results}

This patient series included initially 16 patients, only 13 continued follow up till end of their treatment. The mean age of patients was 32 (range 21 to 55 ). Nine patients were females $(69 \%)$ while 4 were males $(31 \%)$.

All 13 patients complained of low back pain extending to the gluteal region and sometimes to the back of the ipsilateral thigh and/ or groin. Six patients had MRI evidence of lumbar spondylosis and 2 of them already underwent discectomy for lumbar disc herniation with no or minimal improvement. Two patients received local steroid injection in the SIJ region with transient mild improvement. Three patient received radiofrequency ablation of the facet joints and posterior innervation of the SIJ.

The mean time interval from initial complaint till diagnosis was 12 (range: 7-30) weeks. The mean duration on parenteral antibiotics for these 13 patients was 2.6 weeks (18 days) (range 2.1 to 4.9 weeks; 15 34 days). As for post-operative hospital stay, the mean was 1.2 weeks (range 0.7 to 2.1 weeks; 5 - 15 days). The mean duration for total antibiotic treatment till serological test normalization was 7.4 weeks (range 5.7 to 10.6 weeks; 40-74 days).

WBC count was elevated in 5 out of 13 patients, high normal in 6 patients and normal in 2 patients. C-reactive protein and ESR levels were at least double the normal in all 13 patients.

Radiological changes in the form of SIJ destruction/sclerosis (x-ray, CT) were evident in 10 of the 13 patients. MRI evidence of SIJ infection was noted on STIR sequence images in all 13 patients. The pathogens isolated were Staph. aureus in 69\% (9 patients), streptococci in 23\% (3 patients), and E.coli in 7\% (1 patient).

Mean improvement in VAS at end of treatment was 5.5 (range 3 to 6.5), while mean improvement in ODI at end of treatment was 64 (range 44 to 78 ). Four patients continued to use Analgesics despite treatment and normalization of laboratory tests. Still, they were satisfied with the treatment; their mean VAS and ODI improvement without analgesics were 3.5 and 50 respectively.

Complications occurred in 4 patients (30\%) and included, pelvic abscess formation, ilio-psoas abscess formation, posterior subcutaneous abscess, septicemia, SIJ instability.

\section{Discussion}

Pyogenic infection of the SIJ is relatively uncommon, with most descriptions being case reports. ${ }^{1,11}$ It is most often associated with other predisposing factors as traumatic events, skin or gynecologic infections, and IV drug abuse. But, $44 \%$ of patients have no identifiable predisposing factors. In most of the cases the route of infection is unclear, but haematogenous spread from a distant or nearby source, direct spread from an adjacent organ, or postoperative or post-injection infection has been reported as possible causes. ${ }^{12}$

The natural history of an untreated pyogenic SIJ infection starts with radiographic joint space widening, with later progression to blurring of the subchondral bone. The cartilage lining of the sacrum is thicker, an anatomical fact that explains why the iliac side is affected earlier. In some patients, spontaneous fusion may be the end result. ${ }^{6,7}$

The clinical presentation of SIJ infection usually mimic and is confused with that of other more benign pathological conditions of the surrounding musculo-skeletal structures, namely the lumbosacral spine and the hip joints. The distribution of pain in the lower back region, its sometimes diffuse nature, and its extension to the gluteal region, posterior thigh, and/or groin is usually attributed to degenerative disorders of the lumbosacral spine or the hip joints. This is in many instances falsely confirmed by an X-ray, MRI or a CT scan showing herniated discs and/or arthritic facets. Some patients may even undergo unnecessary surgeries with transient, minimal or no improvement. And as SIJ infection is a rare clinical diagnosis, it is often missed.

Certain diagnostic criteria should raise the suspicion about the true diagnosis; swelling or localized tenderness over the SIJ (rather than the Lumbar spine), positive pelvic compression tests, Patrick's test, presence of fever, anorexia, or presence of severe or mechanical symptoms in the absence of obvious radiological signs of lumbosacral instability.

The prolonged history of failed medical/conservative treatment should also direct the physician to suspect the presence of infection whether in the SIJ or the lumbar spine, and therefore order serological testing. A pre-existing nearby (Urinary tract) or even a distant (respiratory or skin) septic focus, an immune-compromised status of the patient (uncontrolled diabetes, immunosuppressive therapy, previous or current malignancy, organ transplantation) can be predisposing factors to infection. IV drug abuse and previous minor pelvic trauma have been also reported in some patients diagnosed with pyogenic SIJ infection. ${ }^{1,3}$ 
Physical examination will usually reveal positive straight leg raising, posterior compression and Patrick's tests. Fever is usually of low grade. Elevated ESR and C-reactive protein together with an elevated leucocyte count are usually present and confirm suspected infection. Blood culture is only positive in about $50 \%$ of patients. 3 Plain radiographs are usually inconclusive especially at early stages. But, MRI is sensitive at early detection and is even more specific than bone scintigraphy. ${ }^{13}$

MRI represents the most sensitive and specific method in early diagnosis of infectious sacroiliitis. The short tau inversion recovery sequence (STIR) was found to be more effective than the T1 contrast-enhanced sequence, particularly in delineating all findings of the SI joint inflammation and allowing for the early detection of septic sacroiliitis. ${ }^{2}$

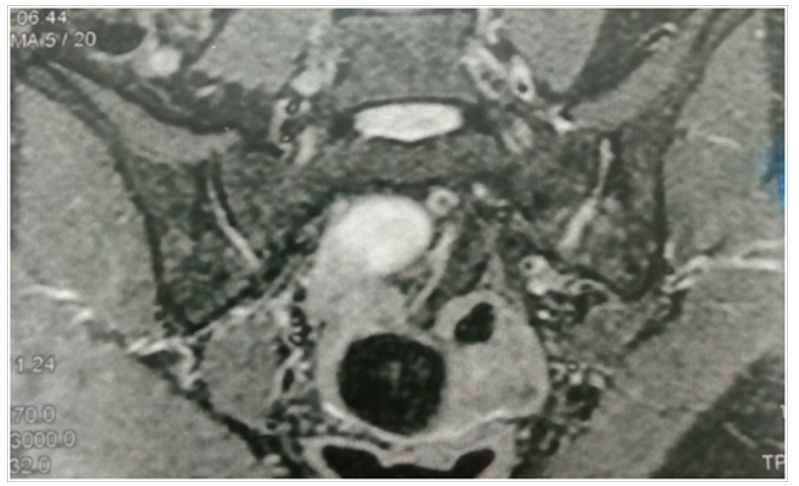

Figure I widening of left SIJ space on coronal fat suppression T2 with high signal intensity denoting pus more on the left side joint.

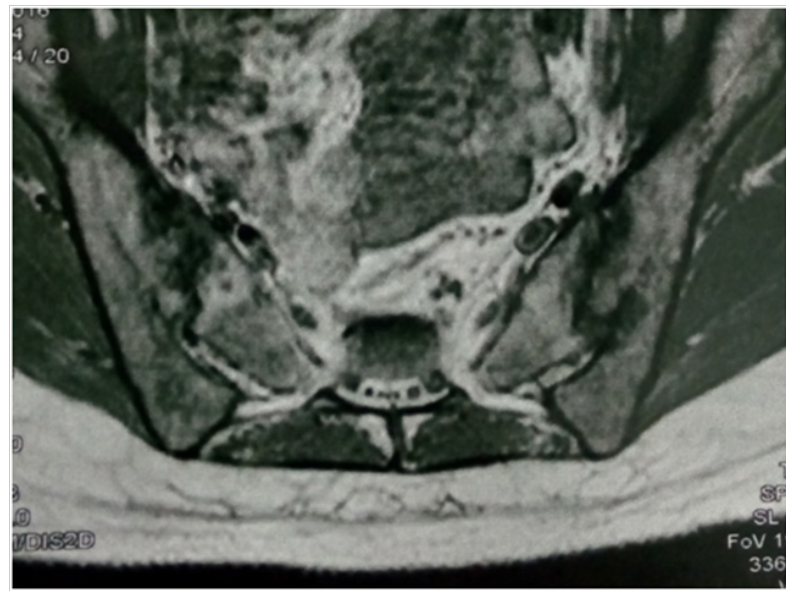

Figure 2 left SIJ showing low signal intensity (edema and suppuration) and irregular joint outline (denoting destruction) on TI MRI.

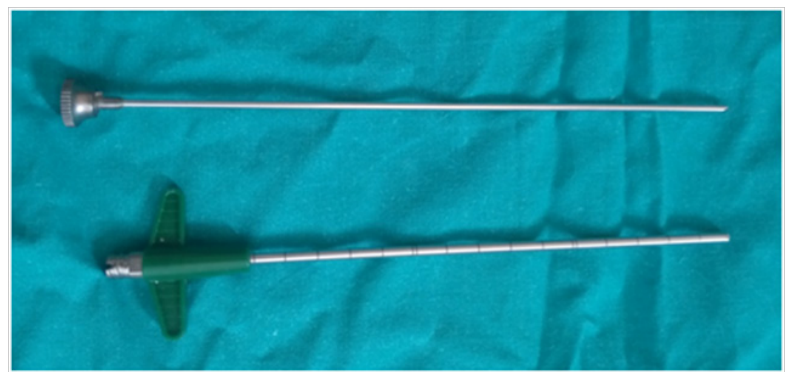

Figure 3 Drainage cannula and trocar used to drain the SIJ (only the cannula was introduced over the inserted guide wire).

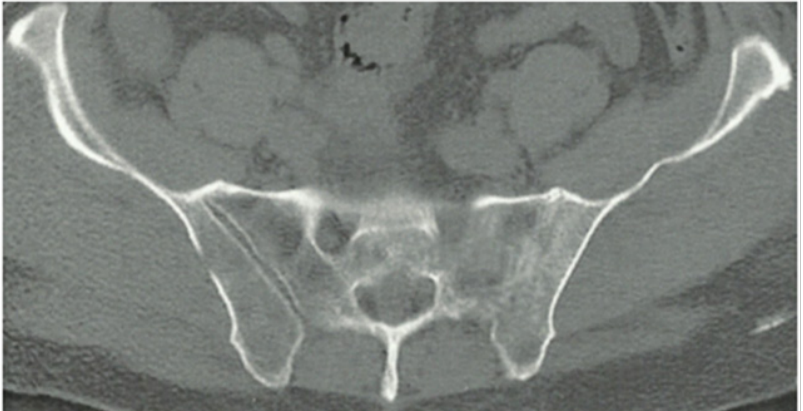

Figure 4 Post-drainage solid union of SIJ (3 months post-op).

Some authors reported good result after bed rest and antibiotic therapy for some weeks1, which might be appropriate for acute cases diagnosed early before chronicity and joint/bone destruction. Surgical drainage and debridement with appropriate antibiotics however is deemed necessary for patients with delayed diagnosis and evident abscess formation or bone destruction. ${ }^{4,14,15}$

Since most patients included in this study were of late diagnosis, with subsequent abscess formation and bone destruction, the role of antibiotic therapy alone was of no value without surgical drainage. And while some authors stated that abscess drainage, sequestrectomy and obtaining tissue for histological/bacterial examination usually requires extensive surgery with considerable morbidity and a long period of rehabilitation. ${ }^{1,14,16}$ This study suggests that minimally invasive drainage procedures can be as effective in obtaining accurate bacterial diagnosis, rapid symptom relief and local control of infection with minimal local and systemic morbidity, and short period of rehabilitation.

Giannoudis and Tsiridis described a case report were they drained the SIJ infection through a lateral transgluteal percutaneous route. The sacroiliac joint is L-shaped and care is required when introducing the guide wire. The safe zone lies between the alar cortex superoanteriorly and the sacral neural foramen posteriorly. They used pelvic inlet and outlet views to insert the guide wire just lateral to the sacral 1 body (S1) foramen. A lateral view was used to direct the wire into the sacroiliac joint then a dynamic hip screw-triple drill reamer was used to debride the joint and to create a channel for drainage. The body of sacral 2 (S2) was drilled over a second guide wire using the $6.5 \mathrm{~mm}$ cannulated drill. ${ }^{9}$

\section{Complications}

Unusual extensions have also been reported; Dangles reported 2 cases in which abscesses dissected through fascial planes into the lower leg and also proximally into the paraspinals in one case with abscess formation. ${ }^{15}$ In our patient series complications occurred in 4 patients $(30 \%)$. One patient had a pelvic abscess formation and ilio-psoas abscess formation which needed anterior drainage and debridement. One patient had a posterior subcutaneous abscess that was drained through a separate incision over the sacro-iliac joint. Two patients had septicemia which subsided after drainage and anti-biotic treatment.

\section{Conclusion}

The lack of high index of suspicion as well as the rare frequency of pyogenic SIJ infection has resulted in delayed diagnoses and sometimes unnecessary interventional or surgical procedures. Risk factor considerations, local examination of SIJ as well as serological testing are keys to proper early diagnosis. Clinical suspicion can be 
confirmed by MRI. CT guided aspiration and culture and sensitivity results will guide adequate antimicrobial treatment. When conservative treatment fails or presentation is late with abscess formation and or bone destruction, minimally invasive image guided drainage and irrigation can be very useful and will have minimal morbidity.

\section{Ethical approval}

Ethical approval: All procedures performed in studies involving human participants were in accordance with the ethical standards of the institutional and/or national research committee and with the 1964 Helsinki declaration and its later amendments or comparable ethical standards and the manuscript is approved from research ethical committee (REC), Faculty of medicine, Ain Shams University (FMASU).

The FMASU REC is organized and operated according to guidelines of the International Council on Harmonization (ICH) and the Islamic Organization for Medical Sciences (IOMS), the United States Office for Human Research Protections ) and the United States Code of Federal Regulations and operates under Federal Wide Assurance No. FWA 000017585.

\section{Acknowledgments}

None.

\section{Conflicts of interest}

None.

\section{References}

1. McGaughey I. Pyogenic infection of the sacroiliac joint. Aust $N Z J$ Surg. 1996;66(5):282-286.

2. Bellussi A, Busi Rizzi E, Schinina V, et al. STIR sequence in infectious sacroiliitis in three patients. Clin Imaging. 2002;26(3):212-215.

3. Hodgson BF. Pyogenic sacroiliac joint infection. Clin Orthop. 1989;246:146-149.
4. Schwartz CM, Demos TC, Wehner JM. Osteomyelitis of the sacrum as the initial manifestation of Crohn's disease. Clin Orthop. 1987;222:181-185.

5. Reilly JP, Gross RH, Emans JB, et al. Disorders of the sacro-iliac joint in children. J Bone Joint Surg Am. 1988;70(1):31-40.

6. Osman AA, Govender S. Septic sacroiliitis. Clin Orthop Relat Res. 1995;(313):214-219.

7. Aprin H, Turen C. Pyogenic sacroiliitis in children. Clin Orthop. 1993;287:98-106.

8. Govender S, Chotai PR. Salmonella osteitis and septic arthritis. $J$ Bone Joint Surg Br. 1990;72(3):504-506.

9. Giannoudis PV, Tsiridis E. A minimally-invasive technique for the treatment of pyogenic sacroiliitis. $J$ Bone Joint Surg Br. 2007;89(1):112-114.

10. Magee DJ. Orthopaedic physical assessment. (3rd edn), WB Saunders, Philadelphia, USA. 1997.

11. Abbott GT, Carty H. Pyogenic sacroiliitis, the missed diagnosis? $\mathrm{Br} J$ Radiol. 1993;66(782):120-122.

12. Zimmermann B, Mikolich DJ, Lally EV. Septic sacroiliitis. Semin Arthritis Rheum. 1996;26(3):592-604.

13. Blum U, Buitrago-Tellez C, Mundinger A, et al. Magnetic resonance imaging (MRI) for detection of active sacroiliitis - a prospective study comparing conventional radiography, scintigraphy, and contrast enhanced MRI. J Rheumatol. 1996;23(12):2107-2015.

14. De Miguel J, Collazos J, Ayarza R, et al. Pubic osteomyelitis due to Pseudomonas aeruginosa. Clin Infect Dis. 1993;17(3):515-516.

15. Dangles CJ. Two unusual presentations of pyogenic sacroiliitis. Orthop Rev. 1987;16(5):327-330.

16. Attarian DE. Septic sacroiliitis: the overlooked diagnosis. J South Orthop Assoc. 2001;10(1):57-60. 